\title{
Extracorporeal Circulatory/Life Support: An Update
}

\author{
Justiaan L. C. Swanevelder ${ }^{1}$ Richard K. Firmin ${ }^{2}$ \\ ${ }^{1}$ Department of Anaesthesia and Perioperative Medicine, University \\ of Cape Town, Groote Schuur and Red Cross War Memorial \\ Children's Hospitals, Cape Town, South Africa \\ 2Department of Cardiothoracic Surgery, Heart Link Extracorporeal \\ Membrane Oxygenation (ECMO) Centre, University Hospitals of \\ Leicester NHS Trust, Leicester, United Kingdom \\ J Card Crit Care TSS 2017;1:65-71
}

\begin{abstract}
Address for correspondence Justiaan L. C. Swanevelder, Department of Anaesthesia and Perioperative Medicine, University of Cape Town, Groote Schuur and Red Cross War Memorial Children's Hospitals, Cape Town, South Africa (e-mail: justiaan.swanevelder@uct.ac.za).
\end{abstract}
Abstract
Keywords
- acute lung injury
- extracorporeal mem- brane oxygenation
- extracorporeal life support

The evolution of extracorporeal life support technology has added a new advanced dimension to intensive care management of acute cardiac and/or respiratory failure in neonatal, pediatric, and adult patients who fail conventional treatment. ECMO has been a controversial subject within the intensive care community for many years. Perceptions have, however, changed positively over the past decade due to a need for improved management of these groups of patients, technological advances, and evolving evidence. As is common with many emerging therapies, its optimal use is currently not fully backed by quality evidence.

\section{Introduction}

The cardiorespiratory system is responsible for maintenance of adequate oxygen delivery $\left(\mathrm{DO}_{2}\right)$ to the cells to meet ongoing demands during health and disease conditions. Under normal conditions, the metabolic rate of cells controls $\mathrm{DO}_{2}$; therefore, it is a demand-driven process. Oxygen moves down a partial pressure gradient from the atmosphere through the respiratory tract, alveolar gas, arterial blood, and systemic capillaries into the cell. It reaches its lowest level (5-15 mm $\mathrm{Hg}$ ) inside the mitochondria, where it is utilized. This oxygen delivery or $\mathrm{DO}_{2}$ gradient is called the oxygen cascade.

\section{Physiology/Pathophysiology}

Although it is outside the scope of this review to discuss physiology, pathophysiology, and physics in detail, it is assumed that the critical care physician will have a good understanding of the following principles and equations, and where they fit in:

- $\mathrm{VA}=\mathrm{VCO}_{2} / \mathrm{PaCO}_{2} \times \mathrm{K}$

- $\mathrm{PIO}_{2}=\mathrm{FiO}_{2} \times\left(\mathrm{PB}-\mathrm{PH}_{2} \mathrm{O}\right)\left(\mathrm{PIO}_{2}=20.93 / 100 \times[760-47]=\right.$ $149 \mathrm{~mm} \mathrm{Hg}$ )

- Alveolar gas equation: $\mathrm{PaO}_{2}=\mathrm{Pio}_{2}-\mathrm{PACO}_{2} / \mathrm{R}+\mathrm{F}$

- ( $\mathrm{F}$ is a small correction fraction and $\mathrm{R}=\mathrm{Vco}_{2} / \mathrm{Vo}_{2}$, also called respiratory quotient, with a normal value of 0.8 )
- Fick's law of diffusion: Vgas $=\mathrm{A} / \mathrm{T} \times \mathrm{D} \times(\mathrm{P} 1-\mathrm{P} 2)$ (D = sol $/ \mathrm{mw})$

- Shunt equation: $\mathrm{Q}_{\mathrm{S}} / \mathrm{Q}_{\mathrm{T}}=\mathrm{CCO}_{2}-\mathrm{CaO}_{2} / \mathrm{CCO}_{2}-\mathrm{CvO}_{2}$

- Ventilation-perfusion mismatch, venous admixture, and Henry's law

- The structure of hemoglobin and how it carries oxygen. $\mathrm{O}_{2} \mathrm{Hb}$-disscociation curve, $\mathrm{P} 50$

- Oxygen content of blood: $\mathrm{CAO}_{2}=(1.39 \times \mathrm{Hb} \times$ Saturation/100) + $\left(0.003 \times \mathrm{PaO}_{2}\right)$

- Bohr effect, Hamburger effect

- Oxygen delivery/oxygen flux: $\mathrm{Do}_{2}=\mathrm{CO} \times \mathrm{CaO}_{2}$

- The Fick principle, oxygen consumption: $\mathrm{Vo}_{2}=\mathrm{CO} \times$ $\left(\mathrm{CaO}_{2}-\mathrm{CvO}_{2}\right)$

- Murray acute lung injury scoring system, Berlin ALI definitions

- Oxygenation index (OI) $=$ Mean airway pressure $\times \mathrm{FiO}_{2} / \mathrm{PaO}_{2}$

- $\mathrm{PaO}_{2} / \mathrm{FIO}_{2}$

- Hagen-Poiseuille equation: $V=P \pi r^{4}$

$$
\begin{gathered}
8 \mathrm{nl} \mathrm{P}=\mathrm{Q} \times \mathrm{R} \\
\mathrm{R}=8 \mathrm{nl} \\
\pi \mathrm{r}^{4}
\end{gathered}
$$

- A good understanding of evidence-based principles of ICU, such as protective ventilation strategies, fluid management, prone positioning, high-frequency oscillatory ventilation (HFOV), etc.
DOI https://doi.org/ 10.1055/s-0038-1626674 ISSN 2457-0206.
Copyright $\odot 2017$ Official Publication of The Simulation Society (TSS), accredited by International Society of Cardiovascular Ultrasound (ISCU)
License terms

(9) (1) $\Theta \circledast$ 


\section{Extracorporeal Life Support}

The evolution of extracorporeal life support (ECLS) technology has added a new advanced dimension to intensive care management of acute cardiac and/or respiratory failure in neonatal, pediatric, and adult patients who fail conventional treatment. ${ }^{1}$ ECLS also complements cardiac surgical and cardiology procedures, implantation of long-term mechanical cardiac assist devices, heart and lung transplantation, and cardiopulmonary resuscitation.

This review summarizes the available ECLS options and provides insights into the principles (physiology and physics) and practice of these techniques. One should emphasize that, as is common with many emerging therapies, their optimal use is currently not yet fully backed by quality evidence. ${ }^{2}$

The recent upsurge in interest in this subject has been precipitated by a coincidence of several factors:

- Technological improvements in oxygenator, pump, and cannula design.

- The CESAR (conventional ventilatory support vs. extracorporeal membrane oxygenation for severe adult respiratory failure) trial of adult ECMO. ${ }^{3}$

- A worldwide H1N1 influenza type A pandemic. This pandemic produced a significant cohort of young, critically ill patients despite the mortality for the older patients actually being lower than usual. ${ }^{4}$

\section{History and Evidence}

ECMO is not a novel therapy, with the first adult case being treated in 1972 and the first successful neonate in $1975 .{ }^{5}$ At that stage, ECMO was based on a modification of the cardiopulmonary bypass machine. From 1977, Trahanas and colleagues investigated ECMO further. ${ }^{6}$ They used a lung protective mechanical ventilation strategy known as lowfrequency positive-pressure ventilation (LFPPV) with ECMO. It consisted of a low tidal volume, low frequency, and low peak inspiratory pressure. The aim of this technique was to prevent any further damage to already diseased lungs.

For more than 30 years, ECMO has been a controversial subject within the intensive care community, and for most of that time has only been practiced within a few specialist centers worldwide, usually concentrating on neonatal and pediatric patients. The enthusiasm for adult ECMO after the $1979 \mathrm{NIH},{ }^{7}$ and subsequent studies by Morris et $\mathrm{al}^{8}$ was not much, and only a handful of centers continued to offer it worldwide. Neither of these studies is, however, relevant to current practice, for many reasons related to changes in both ECMO and conventional care management (see later). Many other observational studies have demonstrated the benefits of ECMO. ${ }^{5,9}$

One of the earlier enthusiastic units, The Heart Link ECMO Centre in Leicester, United Kingdom (UK), was involved in two major ECMO trials. The first was the UK Collaborative Trial for Neonate Respiratory Failure, which showed a clear benefit for transfer of critically ill infants from a regional neonatal center to an ECMO center to receive ECMO support. ${ }^{10}$
In the UK, this led to a national four-center neonatal ECMO service providing for the UK as a whole. It has functioned successfully for over 20 years and is ongoing.

The second was the CESAR trial for adult respiratory failure, which randomized patients from established intensive care units to either continuing conventional intensive care in the original hospital or transfer to a single specialist ECMO center (Glenfield Hospital, Leicester, United Kingdom). ${ }^{3}$ This trial reported a statistical benefit for transferring patients with a Murray score of more than 3.0 for ECMO. There were, however, confounding factors with respect to the number of successful conventionally treated patients in the ECMO arm, and a significant mortality during transfer of patients, which had not been a feature of earlier practice.

An important feature of both UK trials was that the endpoint was not survival. Instead, it was intact survival after an interval. In case of the UK Collaborative Neonatal Trial, it was 1-year intact survival based on neurological assessment. In case of the CESAR trial, it was of functional independence at 6 months after treatment. An important, but often unrecognized, aspect of ECMO support is that the quality of survivors at all ages is excellent and that there is little long-term functional disability. Because they are usually young and otherwise healthy before their severe illness, after recovery they go on to have a normal productive life in society. ${ }^{11}$

Coinciding with publication of the CESAR trial was the H1N1 influenza A pandemic, which behaved in an unusual way. Instead of causing major mortality in older patients, particularly those with preexisting respiratory disease, it produced a small number of severely compromised young adults. Typically, these patients were between the ages of 18 and 35, and often obese and/or pregnant. This was first apparent in Australasia where there was an acute demand for ECMO beds over a 3-month period. ${ }^{4}$ When the pandemic reached the Northern Hemisphere, the pattern of infection was of a "slow burn"; that is, a similar number of patients were treated, but over a longer period of time. The Northern Hemisphere had a further hit from H1N1 in the winter of 2011/2012, which had a similar pattern to the Australasian experience in 2010. ${ }^{12}$ Unlike in the CESAR trial, the circuits used almost exclusively included centrifugal pumps with polymethylpentene (PMP) oxygenators.

Out of the H1N1 pandemic came some case-control studies with prospectively controlled data. ${ }^{13}$ As has been pointed out by the authors, these showed significant benefit from accessing ECMO. Although case-control studies have their limitations, experts believe that interpreted together with the CESAR trial data, there is sufficient information to recommend ECMO for some carefully selected adult patients with acute lung injury. During this period of emergency, coordination between experienced and other centers both in the Northern and Southern Hemispheres showed the benefit of experience in case selection and patient management. The other development from the H1N1 outbreak was the much more frequent transfer between centers of patients on ECMO. New technology makes this much easier and safer, and can make for a hub-and-spoke approach, dependent on the geography and health care system involved. ${ }^{2}$ 
The published Xtravent study ${ }^{14}$ confirmed that hypoxemic patients treated with an ultra-protective ventilation strategy (tidal volume: $2-3 \mathrm{~mL} / \mathrm{kg}$ ) have a significantly shorter mechanical ventilation period. This suggests that early initiation of ECMO is important in the patient with critical acute lung injury. Another aspect that may improve results is the concept of "awake ECMO."15 A good example would be a patient with status asthmaticus or acute exacerbation of chronic bronchitis. "Awake ECMO" allows for earlier extubation and mobilization, with markedly decreased complications.

The design and results of the small number of randomized ECMO trials have been controversial and met with criticism. The reality is that such trials are ethically and practically difficult to design, expensive, and time consuming. Given the type of patient being studied, it is also likely that the results may show confounding factors. It is unrealistic to expect any further meaningful data derived from randomized ECMO trials to guide decision making in the future. There is presently sufficient data to suggest that any new trial would have to involve an option for crossover to justify ethical approval. ${ }^{2}$ This is also true for the French ELOIA (Extracorporeal Membrane Oxygenation for Severe Acute Respiratory Distress Syndrome) study. ${ }^{16,17}$ Thus any future data accruing from randomized trials may have major built-in limitations. Individual case series of adult patients with acute respiratory failure managed with ECMO, conducted in the setting of ongoing technological advances, should guide future management protocols.

In the UK, the response to the CESAR trial and the H1NI flu pandemic has been to commission a five-center adult ECMO service in addition to the four-center neonatal and pediatric ECMO service. This is now operating on a regional basis, with transport of patients on ECMO as part of the contract. In the United States and some countries in Europe (e.g., Germany), there has been no national or regional coordination of ECMO services. It is rather driven by institutional enthusiasm.

From the available experience and literature, there is now sufficient evidence to support selective use of ECMO in specific clinical situations, provided the centers offering the service have done the necessary planning, preparation, and training. ${ }^{18}$

\section{Guidelines}

According to the ELSO Guidelines, ${ }^{18}$ the indications where there is evidence to support include:

- Severe neonatal respiratory failure refractory to medical management (e.g., meconium aspiration).

- Support for severe acute respiratory failure in older children and adults with a potentially reversible cause, not responsive to optimal conventional management along the guidelines recommended (However, peri-resuscitation iatrogenic drowning/fluid overload of such patients is a depressingly frequent event.). A useful severity guide for smaller children is an OI of $>40$, and for larger patients a Murray Score of $>3.0$.

- Support for cardiorespiratory failure in patients postsurgery for congenital heart disease.
- Bridge to heart, lung, and heart-lung transplantation. ${ }^{15}$

- Support for reversible right-sided heart failure in acute pulmonary embolism, pulmonary embolectomy, and postoperative pulmonary hypertension in acquired heart disease.

Beyond these general considerations, one should not be too prescriptive. Key to the assessment is potential reversibility. Each case should be judged on its merits, as case selection not only depends on patient factors but also the experience and expertise of the center offering treatment. Clearly, patients with specific contraindications such as severe neurological injury, disseminated malignancy, and severe chronic lung disease should be excluded. However, beyond that, individualized assessment needs to be made. In practice, there will be uncertainties, not least because the most common indication in adults is pneumonia. The most difficult group of patients to assess is that with treatable malignancies, such as leukemia, when they may or may not be salvageable depending on the stage of the disease, its treatment, and the ability to treat underlying infection. For example, varicella in a lymphoma patient will usually be treatable, but not cytomegalovirus (CMV) infection in a bone marrow transplant patient. Other challenging decisions are patients with Pneumocystis pneumonia or HIV/AIDS. These are often young patients with single-organ failure who, should they recover, may have reasonable survival rates, provided that they are compliant with their treatment. Similarly, an experienced center might be able to manage a patient with acute trauma or sepsis in which an inexperienced one may end up with uncontrollable hemorrhage. ${ }^{19}$

In general, if a disease process has a specific treatment or is in any case self-limiting, the patient should be offered support. In the recent Berlin definition of ARDS, ECMO is included in the treatment algorithm of severe ARDS. ${ }^{20}$

\section{Technological Improvements}

Until recently, around 2007, ECMO was generally practiced using a traditional roller pump controlled by a bladder box and a solid silicone membrane oxygenator. It was soon obvious that achieving an acceptable standard using these circuits was difficult and time consuming, and occasional ECMO was to be avoided. Because of the limited demand for long-term use devices, there was little industry research into improving technology. However, when the improvements started to appear, the developments came quickly:

- The introduction of PMP hollow fiber oxygenators. These were long lasting and had a much lower resistance to blood flow than earlier devices. These new PMP oxygenators had no protein leakage over time, which did occur and compromised durability with the previous generation of membranes.

- Highly efficient and durable magnetically operated centrifugal pumps were developed, which were also in use as ventricular assist devices (VADs) and much better suited to the new low-resistance gas exchange devices. 
- The development of purpose built, Silastic, wire-reinforced double-lumen cannulae (e.g., AVALON, MAQUET) with low recirculation characteristics. This final component simplifies cannulation and makes ECMO much more attractive, particularly for adults.

The combination of these improvements makes for a circuit that is durable and efficient, requiring much less bedside interaction than its predecessor. The new double-lumen cannula allows for single catheter insertion, therefore simplifying initiation and improving the efficiency of venovenous ECMO (VV-ECMO). It is also a circuit, which will not blow apart as there is no occlusive roller pump, although air entrainment and embolization can still be a complication. Superficially, therefore, it appears much simpler and safer, and thus many are being tempted to use it without engaging in the necessary training, development of management protocols, and planning necessary for providing this prolonged circulatory support. A major complication for inexperienced users is the occurrence of hemorrhage, often arising from something as simple as an intercostal chest drain insertion. Experience and understanding are required, both to prevent and manage such an event, so that it does not precipitate an untenable clinical situation.

\section{Mode of Extracorporeal Life Support}

The mode of ECMO is an important consideration and should be selected for the need of the specific patient. Available ECLS therapies provide a range of options to the multidisciplinary teams that are involved in the time-critical care of these complex patients:

- Venoarterial ECMO (VA-ECMO) provides mechanical circulatory support in a traditional central/peripheral fashion or in a temporary ventricular assist device configuration. This may stabilize patients with decompensated cardiac failure who have evidence of end-organ dysfunction, allowing time for recovery, decision making, and bridging to implantation of a long-term mechanical circulatory support device and occasionally heart transplantation-circulation support, oxygenation, and $\mathrm{CO}_{2}$ removal.

- Venovenous ECMO (VV-ECMO) can provide complete respiratory support-oxygenation and $\mathrm{CO}_{2}$ removal.

- Extracorporeal carbon dioxide removal $\left(E C_{2} R\right)$ facilitates protective lung ventilation and provides only partial respiratory support- $\mathrm{CO}_{2}$ removal only, not much oxygenation.

\section{Venoarterial Extracorporeal Membrane Oxygenation}

VA-ECMO is used when the patient needs circulatory support in addition to respiratory support/oxygenation. ${ }^{21}$ When used for respiratory failure, VA-ECMO is reserved for those situations when there is additional right heart failure, in cases of pneumonia and conditions such as pulmonary embolism or postoperative pulmonary hypertension. VA-ECMO is not synonymous with cardiopulmonary bypass (CPB), and it does not necessarily produce better gas exchange than VV-ECMO for respiratory failure. In VA-ECMO, the heart is normally filled (often overfilled) and ejecting, whereas that is not the case during CPB, in which the heart is empty.

Arterial cannulation is usually an open surgical procedure done under general anesthesia. Venous drainage access is obtained by cannulation of the internal jugular or femoral vein. After passing through the circuit, pump, and oxygenator, the oxygenated blood is typically returned via the femoral artery, axillary artery, right common carotid artery, or even the aorta through an open transthoracic approach. Femoral artery cannulation results in a patient with one very pink opposite leg and abdomen up to the waist, while the head and chest are blue ("Harlequin effect"). Carotid artery cannulation has a risk of air- or thromboembolism. Also, with VA-ECMO, the high returning arterial pressure in the aorta may cause "cardiac stun," as the heart is ejecting against an increased afterload. In VA-ECMO, a gas blender is used in the circuit to control $\mathrm{FIO}_{2}$, whereas this is not the case in VV-ECMO. The lungs can be rested on minimal settings (e.g., bilevel positive airway pressure synchronized intermittent mandatory ventilation [BiPAP/SIMV], $\mathrm{FIO}_{2} 0.21$, peak airway pressures $<20 \mathrm{~mm} \mathrm{Hg}$, positive end-expiratory pressure $<10 \mathrm{~mm} \mathrm{Hg}$, respiratory rate $8-10$ breaths/min).

\section{Venovenous Extracorporeal Membrane Oxygenation}

During VV-ECMO, the advantage is that the whole arterial system, including the coronary arteries, will have similar oxygenation..$^{22}$ Although $100 \%$ oxygen saturation may not be achieved, $>85 \%$ is adequate for survival and achievable even with no lung function. Similar to VA-ECMO, the lungs should be rested on minimal settings (e.g., BiPAP/SIMV, $\mathrm{FIO}_{2}$ 0.21 , peak airway pressures $<20 \mathrm{~mm} \mathrm{Hg}$, positive end-expiratory pressure $<10 \mathrm{~mm} \mathrm{Hg}$, respiratory rate $8-10$ breaths/ min). Cannulation for VV-ECMO is a less complex procedure compared with VA-ECMO. Methods include cut-down, semi-Seldinger (specifically for neonates), and percutaneous techniques. The procedure is done in theater under fluoroscopic and/or echocardiography guidance. ${ }^{23,24}$

Traditionally, two large veins are cannulated for VV-ECMO, for example jugular and femoral veins, bilateral femoral veins, or bilateral saphenous veins. The major disadvantage of femoral-femoral venous cannulation is recirculation, which will affect oxygenation and can be up to $60 \%$ at times. To avoid this, the two cannulas should be far apart $(>30 \mathrm{~cm})$, for example, draining blood from the femoral vein and after passing through the oxygenator, returning it to the right atrium. A mechanical pump is incorporated in the extracorporeal circuit, and a flow rate of 20 to $30 \%$ of the cardiac output is maintained. Pump flow rate will dictate oxygenation of blood, whereas sweep gas flow affects $\mathrm{CO}_{2}$ removal. As mentioned above, an exciting technological advance in VV-ECMO 
is cannulation of a single vein (usually superior vena cava [SVC]) with a double-lumen catheter. This allows drainage from the SVC and inferior vena cava (IVC) with return flow to the right atrium, all with one cannula. This technology has made cannulation much safer and the modality accessible to more critical care units.

\section{Extracorporeal Carbon Dioxide Removal}

Extracorporeal $\mathrm{CO}_{2}$ removal $\left(\mathrm{ECCO}_{2} \mathrm{R}\right)$ is used for patients with hypercarbia causing respiratory acidosis, refractory to conventional mechanical ventilation. It can be done in two ways: either with VV-ECMO or the arteriovenous pumpless extracorporeal lung assist (PECLA) system. ${ }^{25}$ The predominant feature here is low blood flow and high sweep gas flow, allowing efficient $\mathrm{CO}_{2}$ removal and rest of the sick lung parenchyma. Elimination of carbon dioxide is more effective than oxygenation. The lower blood flow allows smaller venous drainage cannulas to be used, which will decrease complications. Using a smaller cannula may also limit limb ischemia. The PECLA devices (Novalung) have only an oxygenator but no pump in the circuit, and blood flow depends on the patient's own cardiac output. Complications (e.g., limb ischemia) with the PECLA device placement have been quite common and usually relate to the too low positioning of a rigid arterial cannula.

\section{Anticoagulation}

When initiating extracorporeal circulation, it is necessary to anticoagulate the patient, usually with a bolus of heparin (5,000 units) followed by an infusion. ${ }^{26}$ Monitoring of anticoagulation is done by regular assessment of the activated clotting time (ACT). Because hemorrhage is a big risk with any extracorporeal circuit, it is a usual practice to run a reasonably low ACT of around 150 to 180 seconds. Some units use the activated partial thromboplastin time (aPTT) as a guide to anticoagulation (required 40-120 seconds). In case of an uncontrolled coagulopathy, heparin may even be stopped and the patient evaluated with regular thromboelastograms (TEGs). A good understanding of the management of severe coagulopathy is vital to the success of any ECMO unit ( - Table 1). ${ }^{27}$

\section{Administration of Drugs during Extracorporeal Membrane Oxygenation}

This has been widely investigated. Shekar and colleagues proved that a large amount of opioids, sedatives, and certain antibiotics are sequestrated in the ECMO circuit. ${ }^{28}$ This can have serious effects on patient outcome, if not taken into account. To avoid sequestration, it has been advised that drugs are administered distal to the reservoir, but then the risk for air embolism is higher. It is safer to administer drugs proximal to the reservoir, as the top of the reservoir will serve as an air trap, but the degree of sequestration is negatively influenced.

\section{Cost}

The high cost of ECMO, as described in the CESAR trial, is always argued as a reason why it is not a viable treatment option in developing countries. ${ }^{2}$ Although the circuit is expensive, the largest cost of ECMO lies in staffing the service. CESAR was performed with the previous generation circuits and nurse staffing to patient ratio in the ECMO arm was two-to-one (ECMO specialist and critical care nurse to one patient). This is often referred to as "old ECMO." At the same time, the conventional arm had one-to-one nurse staffing. With modern technology, this is not necessary anymore, and a one-to-one ratio is also possible with an ECMO patient. In addition, costs of the disposable circuits are decreasing, plus blood priming of the circuit can be avoided ("modern ECMO"). The cost during the CESAR trial does therefore not reflect modern practice. In addition, ICU length of stay may be reduced because the more aggressive management allows lung rest, with less ventilator-induced lung injury (VILI) and ventilator-associated pneumonia (VAP). Cost can be put into perspective when comparing a young patient with reversible acute lung injury on ECMO, to a patient with leukemia receiving chemotherapy, radiotherapy, and a bone marrow transplant. $^{29}$

Table 1 Complications associated with $\mathrm{ECMO}^{27}$

\begin{tabular}{|l|l|}
\hline Mechanical complications & Patient-related complications \\
\hline $\begin{array}{l}\text { Oxygenator failure } \\
\text { Tubing/circuit disruption }\end{array}$ & Hemorrhage \\
Pump/heat exchanger malformation & Cannulation sites \\
Clotting of circuit & Vaginal \\
& Intracranial \\
& Neurological complications \\
& Difficulty establishing oral feeding in infant with normal \\
& suck reflex \\
& Severe permanent disability \\
& Catheter-related infections \\
& Additional organ failure \\
& Barotrauma \\
& Limb ischemia \\
& Hemolysis \\
& Damage to vasculature \\
\hline
\end{tabular}

Abbreviations: ECMO, extracorporeal membrane oxygenation; GIT, gastrointestinal tract. 


\section{Future}

In future, it is likely that the use of conventional methods of respiratory support, such as positive-pressure ventilation, may decrease, whereas the use of noninvasive methods of oxygenation, such as extracorporeal circuits may increase. For instance, a young patient with H1N1 viral pneumonia has good lung mechanics, with bad gas exchange and oxygenation. Intubation and positive ventilation may therefore not be the ideal treatment in this patient. ${ }^{30,31}$ Compare this to the polio epidemic where patients had bad lung mechanics and weakness, with preserved lung parenchyma. After all, 30 years ago renal replacement therapy used for renal impairment had a very bad outcome, whereas today it is commonplace. This all depends on further technological advances.

\section{Conclusion}

In conclusion, ECMO should be available for selected cases in advanced health care systems and should be performed in centers that have done the necessary planning, preparation, and training. The exact method of achieving this goal depends on the particular health care system and the balance between public and private providers. In general, neonatal and pediatric ECMO should be available in centers doing large numbers of congenital heart operations ( $>300$ cases per year). The organization for adult postcardiotomy ECMO is more problematic, due to the low turnover of cardiac surgical cases requiring ECMO support. The concept of a specialized ECMO center is central to a successful ECMO program. ECMO should not be encouraged in low-volume centers (less than 20 cases per year), which should rather refer to high-volume centers ideally with mortality rates under $50 \%$, where expertise increases exponentially. Rather than a free-for-all developing, there should be a formal discussion between the public sector, private sector providers, and medical aids about whether ECMO should be supported as a treatment modality, and if so, where and how it would be best provided.

We should not be predicting present outcomes from ECMO on the basis of studies conducted in the distant past. It is likely that future case series of patients treated with high-, medium- or low-flow VV-ECMO will show considerably improved outcomes compared with past studies, because of major improvements in gas exchange devices, circuits, centrifugal pumps, and advanced double-lumen cannulas. It is in everyone's interests, particularly those of our patients, that if ECMO is to be done at all, it is done well and as cost-effectively as possible.

\section{References}

1 Shekar K, Mullany DV, Thomson B, Ziegenfuss M, Platts DG, Fraser JF. Extracorporeal life support devices and strategies for management of acute cardiorespiratory failure in adult patients: a comprehensive review. Crit Care 2014;18(3):219

2 Firmin RK, Swanevelder JLC, Ferreira MM. Extracorporeal membrane oxygenation (ECMO) in South Africa. South Afr J Crit Care 2013;29(1):3-5
3 Peek GJ, Mugford M, Tiruvoipati R, et al; CESAR trial collaboration. Efficacy and economic assessment of conventional ventilatory support versus extracorporeal membrane oxygenation for severe adult respiratory failure (CESAR): a multicentre randomised controlled trial. Lancet 2009;374(9698):1351-1363

4 Davies A, Jones D, Bailey M, et al; Australia and New Zealand Extracorporeal Membrane Oxygenation (ANZ ECMO) Influenza Investigators. Extracorporeal membrane oxygenation for 2009 influenza A (H1N1) acute respiratory distress syndrome. JAMA 2009;302(17):1888-1895

5 Bartlett RH, Roloff DW, Custer JR, Younger JG, Hirschl RB. Extracorporeal life support: the University of Michigan experience. JAMA 2000;283(7):904-908

6 Trahanas JM, Kolobow MA, Hardy MA, Berra L, Zapol WM, Bartlett RH. "Treating Lungs": the scientific contributions of Dr. Theodor Kolobow. ASAIO J 2016;62(2):203-210

7 Zapol WM, Snider MT, Hill JD, et al. Extracorporeal membrane oxygenation in severe acute respiratory failure. A randomized prospective study. JAMA 1979;242(20):2193-2196

8 Morris AH, Wallace CJ, Menlove RL, et al. Randomized clinical trial of pressure-controlled inverse ratio ventilation and extracorporeal $\mathrm{CO}_{2}$ removal for adult respiratory distress syndrome. Am J Respir Crit Care Med 1994;149(2 Pt 1):295-305

9 Gattinoni L, Agostoni A, Pesenti A, et al. Treatment of acute respiratory failure with low-frequency positive-pressure ventilation and extracorporeal removal of $\mathrm{CO} 2$. Lancet 1980;2(8189):292-294

10 UK collaborative ECMO Trail Group. UK collaborative randomised trial of neonatal extracorporeal membrane oxygenation. Lancet 1996;348(9020):75-82

11 McNally H, Bennett CC, Elbourne D, Field DJ; UK Collaborative ECMO Trial Group. United Kingdom collaborative randomized trial of neonatal extracorporeal membrane oxygenation: follow-up to age 7 years. Pediatrics 2006;117(5):e845-e854

12 Pham T, Combes A, Rozé H, et al; REVA Research Network. Extracorporeal membrane oxygenation for pandemic influenza A (H1N1)-induced acute respiratory distress syndrome: a cohort study and propensity-matched analysis. Am J Respir Crit Care Med 2013;187(3):276-285

13 Papadopoulos N, Ahmad AE, Marinos S, Moritz A, Zierer A. Extracorporeal membrane oxygenation for influenza-associated acute respiratory distress syndrome. Thorac Cardiovasc Surg 2013;61(6):516:521

14 Bein T, Weber-Carstens S, Goldmann A, et al. Lower tidal volume strategy $\left(\approx 3 \mathrm{ml} / \mathrm{kg}\right.$ ) combined with extracorporeal $\mathrm{CO}_{2}$ removal versus 'conventional' protective ventilation $(6 \mathrm{ml} / \mathrm{kg})$ in severe ARDS: the prospective randomized Xtravent-study. Intensive Care Med 2013;39(5):847-856

15 Fuehner T, Kuehn C, Hadem J, et al. Extracorporeal membrane oxygenation in awake patients as bridge to lung transplantation. Am J Respir Crit Care Med 2012;185(7):763-768

16 Schmidt M, Hodgson C, Combes A. Extracorporeal gas exchange for acute respiratory failure in adult patients: a systematic review. Crit Care 2015;19(99):99

17 Assistance Publique - Hopitaux de Paris. Extracorporeal Membrane Oxygenation for Severe Acute Respiratory Distress Syndrome (EOLIA). In: ClinicalTrials.gov. 2011. Available at: http:// clinicaltrials.gov/ct2/show/NCT01470703. Accessed Nov 7, 2017

18 Extracorporeal Life Support Organization (ELSO). Patient Specific Supplements to the ELSO General Guidelines. 2009:15-19

19 Schupp M, Swanevelder JL, Peek GJ, Sosnowski AW, Spyt TJ. Postoperative extracorporeal membrane oxygenation for severe intraoperative SIRS $10 \mathrm{~h}$ after multiple trauma. Br J Anaesth 2003;90(1):91-94

20 Ranieri VM, Rubenfeld GD, Thompson BT, et al; ARDS Definition Task Force. Acute respiratory distress syndrome: the Berlin Definition. JAMA 2012;307(23):2526-2533 
21 Rihal CS, Naidu SS, Givertz MM, et al; Society for Cardiovascular Angiography and Interventions (SCAI); Heart Failure Society of America (HFSA); Society of Thoracic Surgeons (STS); American Heart Association (AHA), and American College of Cardiology (ACC). 2015 SCAI/ACC/HFSA/STS clinical expert consensus statement on the use of percutaneous mechanical circulatory support devices in cardiovascular care: endorsed by the American Heart Association, the Cardiological Society of India, and Sociedad Latino Americana de Cardiologia Intervencion; Affirmation of Value by the Canadian Association of Interventional Cardiology-Association Canadienne de Cardiologie d'intervention. J Am Coll Cardiol 2015;65(19):e7-e26

22 Sidebotham D, Allen SJ, McGeorge A, Ibbott N, Willcox T. Venovenous extracorporeal membrane oxygenation in adults: practical aspects of circuits, cannulae, and procedures. J Cardiothorac Vasc Anesth 2012;26(5):893-909

23 Platts DG, Sedgwick JF, Burstow DJ, Mullany DV, Fraser JF. The role of echocardiography in the management of patients supported by extracorporeal membrane oxygenation. J Am Soc Echocardiogr 2012;25(2):131-141

24 Firstenberg MS, Orsinelli DA. ECMO and ECHO: the evolving role of quantitative echocardiography in the management of patients requiring extracorporeal membrane oxygenation. J Am Soc Echocardiogr 2012;25(6):641-643
25 Flörchinger B, Philipp A, Klose A, et al. Pumpless extracorporeal lung assist: a 10-year institutional experience. Ann Thorac Surg 2008;86(2):410-417, discussion 417

26 Esper SA, Levy JH, Waters JH, Welsby IJ. Extracorporeal membrane oxygenation in the adult: a review of anticoagulation monitoring and transfusion. Anesth Analg 2014;118(4):731-743

27 Cheng R, Hachamovitch R, Kittleson M, et al. Complications of extracorporeal membrane oxygenation for treatment of cardiogenic shock and cardiac arrest: a meta-analysis of 1,866 adult patients. Ann Thorac Surg 2014;97(2):610-616

28 Shekar K, Roberts JA, Mcdonald CI, et al. Sequestration of drugs in the circuit may lead to therapeutic failure during extracorporeal membrane oxygenation. Crit Care 2012;16(5):R194

29 Majhail NS, Mau LW, Denzen EM, Arneson TJ. Costs of autologous and allogeneic hematopoietic cell transplantation in the United States: a study using a large national private claims database. Bone Marrow Transplant 2013;48(2):294-300

30 Johnson P, Fröhlich S, Westbrook A. Use of extracorporeal membrane lung assist device (Novalung) in H1N1 patients. J Card Surg 2011;26(4):449-452

31 Muscedere JG, Mullen JB, Gan K, Slutsky AS. Tidal ventilation at low airway pressures can augment lung injury. Am J Respir Crit Care Med 1994;149(5):1327-1334 